\title{
Editorial
}

\section{La Biblioteca de la Universidad de Alcalá: innovación y excelencia}

\section{Dolores Ballesteros Ibáñez}

Directora del Servicio de Biblioteca, Universidad de Alcalá; dolores.ballesteros@uah.es

Permítanme comenzar haciéndoles una breve pregunta, ¿conocen la Biblioteca?. Una pregunta directa para conseguir que casi el total de las respuestas sean afirmativas. Sin embargo y muy probablemente, este conocimiento se ajuste a los indicadores y servicios más visibles, a la vivencia que cada usuario haya tenido en los diferentes puntos de servicio e incluso a lo que la propia Biblioteca haya sido capaz de transmitir de una manera más o menos clara y eficaz.

Y si se reformula la pregunta, ¿conocen todo lo que la Biblioteca puede aportarles a lo largo de su vida universitaria e incluso para su formación posterior?. Entonces el porcentaje y el sentido de las respuestas podría variar y podría evidenciar que se debe de trabajar juntos para que el conocimiento mutuo posibilite la mejora continua.

¿Por qué no hacemos una breve reflexión sobre lo que se ha conseguido?. Desde su creación, paralela a la refundación de la Universidad de Alcalá (UAH) en 1977, la Biblioteca no ha dejado de evolucionar y de adaptarse a un entorno cambiante, marcado principalmente por el avance de la tecnología. Entrado el siglo XXI, el cambio se hizo más evidente. El nuevo modelo educativo y de aprendizaje, los cambios en la comunicación científica y en la investigación, las nuevas herramientas de gestión y descubrimiento de información, la fortaleza de las alianzas internas y externas a la institución, la crisis económica con las consiguientes restricciones presupuestarias, la importancia de la transparencia en la gestión y de la excelencia en la prestación del servicio, entre otros hitos, han marcado una ruta en la que la Biblioteca ha sabido avanzar, ser flexible, innovar y adelantarse a los nuevos retos y necesidades de la comunidad Universitaria y de la sociedad, cumpliendo con su misión.

La Biblioteca como sala de estudio con estanterías con libros y revistas en formato papel evolucionó a la biblioteca electrónica y digital, a la biblioteca como Centro de Recursos para el Aprendizaje y la Investigación (CRAI); de la consulta de los fondos bibliográficos in situ se pasó a la consulta de una extensa colección electrónica desde cualquier lugar y en cualquier momento; del desarrollo de una colección propia y autosuficiente, a la gestión de una colección basada en la evidencia y en el usuario y a una colección compartida; de la soledad en la gestión y en la prestación de servicios al desarrollo de fuertes alianzas; del espacio físico al espacio físico y virtual; de la biblioteca como sala de estudio a la diversificación y flexibilidad de espacios; de los servicios de préstamo de libros y consulta en sala a la prestación de servicios online y acceso a la información a través de la web y de las herramientas de descubrimiento; de la formación de usuarios en el uso de los servicios básicos y de las primeras bases de datos en formato $C D$, a la formación en competencias informacionales, la participación en equipos de innovación docente, el apoyo al investigador en la elaboración y difusión del trabajo científico y en la publicación y los datos de investigación en acceso abierto; en suma, de una Biblioteca local a una Biblioteca en un mundo global donde las sinergias dentro de la institución y las alianzas externas han sido junto a los avances tecnológicos y la profesionalidad del personal que la conforma, el motor del cambio.

Entonces, ¿redescubrimos juntos la Biblioteca?.

La Biblioteca de la UAH se ha consolidado en los últimos 8 años como un servicio de calidad. El Sello 500+ según el modelo de excelencia EFQM (European Foundation for Quality Management) obtenido en diciembre de 2017, certifica el esfuerzo realizado desde que comenzó el arduo camino de la calidad total. Y esto ¿qué significa?. Fundamentalmente que todo lo que se hace tiene una clara 
orientación al usuario final, y a la satisfacción de sus necesidades y expectativas. Por lo tanto, conocerlas, comprender su entorno y adelantarnos a sus expectativas es el horizonte que no debe perderse de vista para poder seguir avanzando, mejorando e innovando. El modelo de gestión excelente permite tener un autoconocimiento de la Biblioteca, gestionar por procesos comunes, evaluar los resultados obtenidos y aplicar medidas de mejora para seguir avanzando. En este contexto, la comunicación con el usuario es vital y absolutamente necesaria, una comunicación que debe de ser fluida y estar sistematizada para que la información fluya en ambas direcciones.

Un criterio importante del modelo de calidad son las alianzas. Para la Biblioteca de la UAH, han sido clave las establecidas principalmente con dos organismos. Por un lado, REBIUN (Red de Bibliotecas Universitarias Españolas), Comisión Sectorial de la Conferencia de Rectores de las Universidades Españolas (CRUE), que posibilita un marco de actuación en base a la cooperación, con unas directrices y una estrategia común para las bibliotecas universitarias y científicas españolas. Por otro, el Consorcio de Universidades de la Comunidad de Madrid y de la UNED para la Cooperación Bibliotecaria (Consorcio Madroño) del que ahora se cumplen 20 años de su nacimiento y del que la Universidad de Alcalá es miembro de pleno derecho. El Consorcio ha sido un elemento dinamizador y garante de éxito para la innovación en los servicios bibliotecarios, siendo el único consorcio español de bibliotecas certificado bajo la norma de calidad ISO 9001. Entre los principales proyectos llevados a cabo destacan: la adquisición consorciada de una excelente colección electrónica de recursos de información en unas condiciones de sostenibilidad económica difícil de alcanzar en solitario, la selección, adquisición e implementación de los sistemas de gestión bibliotecaria que han posibilitado automatizar procesos de gestión y servicios al usuario, el desarrollo del repositorio institucional eBuah incardinado en el Proyecto Madroño e-Ciencia, cofinanciado por la Comunidad de Madrid, el Portal de investigación Investigam y el repositorio de datos científicos eCiencia Datos.

Otro criterio clave del modelo EFQM es la estrategia. Aunque en la Biblioteca se ha trabajado siempre por objetivos, fue a partir de iniciar el camino a la calidad total, cuando esa forma de hacer se sistematiza a través de planes estratégicos que vertebran la actividad y los proyectos de la Biblioteca. Actualmente se está trabajando en el desarrollo del IV Plan Estratégico 2018-2022. Las cuatro líneas estratégicas en las que se enmarcan los objetivos y proyectos del Servicio son: organización y comunicación interna, promover y afianzar las relaciones con los grupos de interés, sostenibilidad y responsabilidad social y tecnología. En 2018, se ha trabajado en varios proyectos de relevancia: La implementación y puesta en producción de la Plataforma de Servicios Bibliotecarios ALMA y de la herramienta de descubrimiento de la información PRIMO, que posibilitan unificar la gestión de los recursos de información y ofrecer al usuario un único punto integrado de acceso a la información; la reestructuración y actualización con un nuevo gestor de contenidos, de la información contenida en la web de la Biblioteca en los apartados de apoyo al aprendizaje y de apoyo a la investigación; y la contribución a la difusión de los datos de investigación en abierto a través del repositorio eCiencia Datos, colaborando en varios proyectos de la Universidad.

¿Y el futuro?

Puede ser apasionante e ilusionante, si se construye entre todos.

El apoyo institucional ha sido y será imprescindible y fundamental para poder alcanzar los logros deseados. Los desafíos de la Universidad serán también los desafíos de la Biblioteca. Como servicio excelente y con clara orientación al usuario, puede y debe apoyar con sus valores, conocimiento, estrategia y la profesionalidad de su plantilla, a la consecución de los objetivos de la propia Institución de la que depende, sin dejar pasar las oportunidades que brinde el entorno cambiante.

En el horizonte hay varios temas de relevancia: la Ciencia Abierta (Open Science), el aprendizaje y la formación a lo largo de la vida, la sostenibilidad en sus tres vertientes (económica, medioambiental y social), la preservación digital, la calidad, la tecnología y los servicios innovadores a los usuarios, la medición del impacto y la transparencia en la gestión.

Para afrontar los nuevos retos, la Biblioteca debe afianzar su modelo de gestión centralizado, con una política, una estrategia, una normativa, y unos procesos comunes, de tal forma que todos los 
usuarios encuentren el mismo apoyo a su actividad de aprendizaje, docencia o/e investigación en cualquiera de los puntos de servicio repartidos entre los tres campus de la Universidad.

Sería deseable que el modelo de Biblioteca como Centro de Recursos para el Aprendizaje y la Investigación (CRAI), centralizando servicios y recursos humanos, convergiendo con otros servicios de la universidad y con los espacios y la tecnología necesaria para satisfacer las demandas y expectativas de los usuarios, se impusiera en un futuro en el Campus Científico Tecnológico y en el Campus de Guadalajara, siguiendo el modelo del CRAI Biblioteca del Campus Histórico de Alcalá, inaugurado en 2014 y que ha supuesto una dinamización de la zona centro de la ciudad, una apertura a la ciudadanía y una diversificación del espacio y de la tecnología, imposibles de alcanzar en las antiguas bibliotecas de facultad. Precisamente resultará fundamental redefinir y flexibilizar los espacios; crear aquellos necesarios para la innovación y colaboración con los usuarios; reestructurar el trabajo en la organización para centrarse en el apoyo al aprendizaje y a la investigación; apostar por la tecnología, planificando y utilizando su potencial para crear nuevos servicios al usuario; establecer canales de comunicación y colaboración con el usuario; potenciar la transparencia, la sostenibilidad y la igualdad; poner en valor la Biblioteca.

Permítanme una afirmación para terminar, conozcamos las posibilidades de apoyo, formación e información que la biblioteca brinda a todos sus usuarios, redescubramos sus recursos y servicios, establezcamos sinergias y entornos de colaboración e integrémosla en nuestro día a día en la vida universitaria.

(C) 2019 por los autores; Esta obra está sujeta a la licencia de Reconocimiento 4.0 Internacional de Creative Commons. Para ver una copia de esta licencia, visite http://creativecommons.org/licenses/by-nc-nd/4.0/. 